Revue des patrimoines

$31 \mid 2017$

Patrimoines de la santé : essais de définition - enjeux de conservation

\title{
Lutter contre la tuberculose et la mortalité infantile : l'établissement des dispensaires en France (1880-1950)
}

The struggle againts tuberculosis and infant mortality, the establishment of dispensaries in France from 1880 to 1950

\section{Cécile Lestienne}

\section{OpenEdition}

Journals

\section{Édition électronique}

URL : http://journals.openedition.org/insitu/14026

DOI : 10.4000/insitu.14026

ISSN : 1630-7305

\section{Éditeur}

Ministère de la Culture

\section{Référence électronique}

Cécile Lestienne, «Lutter contre la tuberculose et la mortalité infantile : l'établissement des dispensaires en France (1880-1950) », In Situ [En ligne], 31 | 2017, mis en ligne le 21 février 2017, consulté le 10 octobre 2020. URL : http://journals.openedition.org/insitu/14026 ; DOI : https://doi.org/ 10.4000/insitu. 14026

Ce document a été généré automatiquement le 10 octobre 2020

\section{c) (i) $\Theta$}

In Situ Revues des patrimoines est mis à disposition selon les termes de la licence Creative Commons Attribution - Pas d'Utilisation Commerciale - Pas de Modification 4.0 International. 


\title{
Lutter contre la tuberculose et la mortalité infantile : l'établissement des dispensaires en France (1880-1950)
}

\author{
The struggle againts tuberculosis and infant mortality, the establishment of \\ dispensaries in France from 1880 to 1950
}

Cécile Lestienne

1 Étudier et protéger ce type de patrimoine ne va pas sans difficultés aujourd'hui: édifices modestes à l'échelle de l'histoire de l'architecture contemporaine, les dispensaires sont le plus souvent reconvertis et transformés, quand ils ne sont pas détruits. Ce sujet a cependant rencontré un écho particulier en Bourgogne, avec l'inscription au titre des monuments historiques, en 2011, du dispensaire antituberculeux de Montceau-les-Mines ${ }^{1}$. Cet article est donc l'occasion de dresser une première synthèse et de proposer des pistes de réflexion.

2 Les sources relatives à l'histoire de ces bâtiments sont assez éparses. Citons bien entendu les plus courantes: les archives communales et les archives des institutions départementales chargées de mettre en place les dispensaires dans l'Entre-deuxguerres ${ }^{2}$. Concernant l'architecture elle-même, les fonds du centre d'archives de la Cité de l'architecture et du patrimoine (CAPA), et particulièrement le fonds Béton armé Hennebique $^{3}$, offrent un corpus exceptionnel pour traiter de manière transversale un sujet aussi dense géographiquement. Enfin, un essai de synthèse sur un type architectural ne saurait se passer de la base de données du ministère de la Culture et de la Communication, Mérimée, dans laquelle 65 dossiers d'Inventaire mentionnent un dispensaire. Dans de nombreux cas cependant, ces dispensaires ne sont pas étudiés en tant que tels, ou les dossiers concernent ceux qui ont été installés dans des édifices ayant à l'origine une autre fonction. Grâce à ces différentes ressources, un corpus de 30 dispensaires, construits entre 1880 et 1950, a pu être établi pour cette contribution. Pour la bibliographie, peu exhaustive sur le sujet, il faut citer le bel article de synthèse 
écrit par Yvonne Knibiehler ${ }^{4}$ sur les dispensaires anti-tuberculeux. D'une manière générale, on se référera aux ouvrages traitant de l'histoire des sanatoriums et de la puériculture ${ }^{5}$. L'histoire de l'architecture des dispensaires en tant qu'équipement médical et social de proximité reste à écrire.

Un dispensaire peut se définir comme un «établissement médical destiné à donner des consultations gratuites, des médicaments et à vacciner les enfants ${ }^{6} »$. Certains dispensaires présentent néanmoins des usages plus spécifiques, comme les dispensaires dits «antituberculeux», ou encore ceux de l'œuvre "la Goutte de lait »7. Le plus souvent, le dispensaire a tendance à réunir plusieurs fonctions dans un même bâtiment: ainsi trouvera-t-on dans les sources les mentions plus générales de " dispensaire municipal», de " centre médical », de " centre de protection maternelle et infantile ", de "centre d'hygiène sociale ", etc. Leur affirmation à la fin du XIX siècle est issue d'une double préoccupation : la mortalité infantile, toujours plus importante, et les ravages de la tuberculose dans les milieux populaires, la deuxième étant souvent cause de la première. Suite à de nombreuses enquêtes dans les quartiers où réside la population ouvrière, les médecins hygiénistes dénoncent l'insalubrité et les mauvaises conditions de vie qui y règnent. Financés par les communes, les industriels ou les sociétés de secours mutuel, les dispensaires sont destinés au moment de leur création aux plus démunis, souvent dans une situation sanitaire critique, avant d'être ouverts à tous, dans le cadre d'une politique d'hygiène publique. Longtemps dépendants de l'initiative privée, les dispensaires connurent une forte croissance dans l'Entre-deuxguerres pour devenir un véritable équipement public, au même titre que la mairie ou l'école.

\section{L'établissement des dispensaires avant la Première Guerre mondiale : une initiative privée}

\section{Les fronts sanitaires au tournant du XXe siècle}

Dans le dernier tiers du XIX ${ }^{e}$ siècle, les préoccupations sanitaires sont croissantes; un souci d'assainir et d'éduquer le peuple jusque dans son lieu de vie marque particulièrement cette époque ${ }^{8}$. En témoignent la promulgation de la loi Roussel, en 1874, sur la protection de la petite enfance, ou encore la loi de Santé publique, en 1902, qui impose la déclaration de certaines maladies infectieuses, la désinfection du logis des malades et les vaccinations. Dans le cadre de cette prise de conscience, le manque d'équipements publics se fait ressentir. Trois fronts sanitaires se mettent ainsi en place, et poussent à la création de dispensaires dans des quartiers majoritairement ouvriers. On y distingue la lutte contre la syphilis, sur laquelle nous ne pourrons nous attarder, celle contre la mortalité infantile et celle contre la tuberculose.

Ce sont les maladies intestinales, sévissant chez les jeunes enfants des milieux ouvriers, qui entraînent une forte croissance de la mortalité infantile. Une des principales causes est l'alimentation des bébés par du lait non stérilisé. Les mères travaillant, elles ne peuvent allaiter leurs enfants comme il le faudrait, d'après les médecins de l'époque. C'est dans ce contexte qu'est créé en 1894 un premier dispensaire, appelé «Goutte de lait ", par le docteur Léon Dufour, à Fécamp". Deux années auparavant, le docteur Gaston Variot avait lui aussi fondé un dispensaire dans le quartier populaire de Belleville, à Paris (fig. 1) : l'objectif était de soigner les bébés. Ce mouvement fit tache 
d'huile. Des «Gouttes de lait» se créent par la suite un peu partout en France. Leurs fonctions sont tout à la fois médicale, sociale et éducative. Elles encouragent l'allaitement maternel et préparent, pour les mères ne pouvant nourrir leur enfant, un lait de bonne qualité, c'est-à-dire stérilisé. Ainsi, à Montceau-les-Mines, la Société anonyme des mines de Blanzy n'est-elle pas insensible à ce phénomène. Dès 1905, elle établit un service de consultation pour les nourrissons, complété en 1906 par l'institution d'une Goutte de lait ${ }^{10}$. Une seconde Goutte de lait est construite par l'architecte montcellien Marcel Fournier dans la cité des Gautheret en 1925.

Figure 1

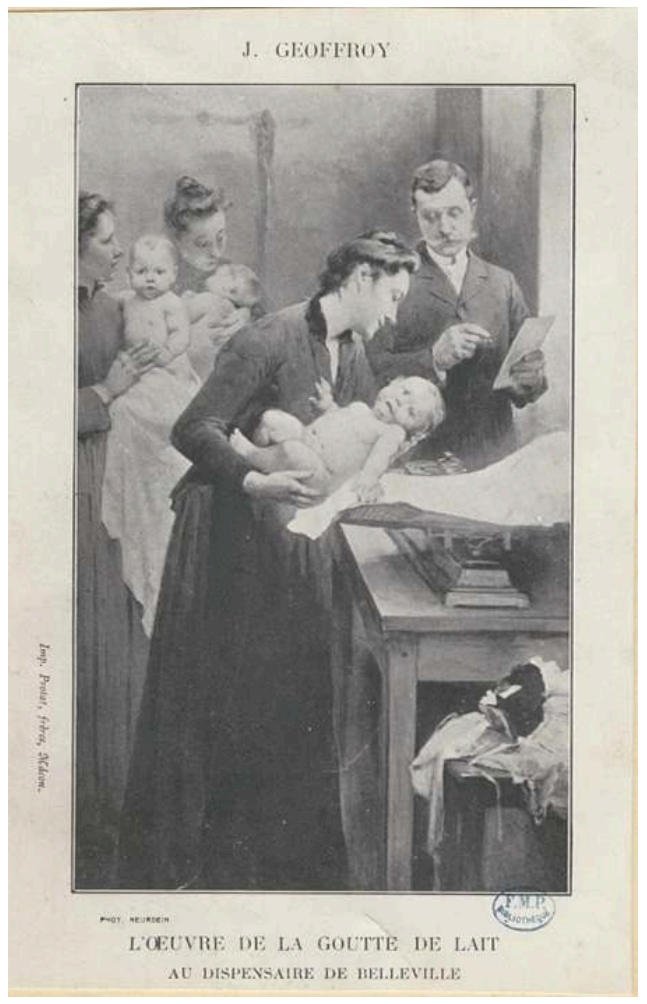

L'Fuvre de la goutte de lait au dispensaire de Belleville. Phot. Neurdein. (C) BIU Santé (Paris).

6 La lutte contre la tuberculose constitue un autre front sanitaire préoccupant. Elle n'est cependant pas vraiment relayée en France avant la Première Guerre mondiale, la contagiosité de cette maladie étant contestée dans le corps médical français durant la deuxième moitié du XIX ${ }^{e}$ siècle. Il faut attendre l'isolation du bacille par Koch en 1882, et la confirmation scientifique du médecin militaire Jean-Antoine Villemin pour que l'Académie de médecine se décide à faire une déclaration officielle en 1889. Aussi, l'action antituberculeuse n'est-elle d'abord le fait que d'initiatives privées dispersées et sans grands moyens ${ }^{11}$. En 1901, Albert Calmette, alors directeur de l'institut Pasteur de Lille et fondateur de l'œeuvre antituberculeuse du Nord, adapte la formule du dispensaire général: au lieu d'accueillir n'importe quel malade, il spécialise un dispensaire dans la lutte contre la tuberculose. Il en fait un instrument non pas de soin, l'hôpital étant mieux équipé, mais de dépistage, de prophylaxie et d'assistance aux tuberculeux ${ }^{12}$. Pour lui, l'action préventive est primordiale, les moyens curatifs des sanatoriums privés étant aléatoires et sélectifs. Ces derniers ne soulageaient en effet, et 
éventuellement ne guérissaient, que les malades déclarés "curables». Les « incurables », qui n'avaient pu bénéficier d'une bonne prévention, étaient alors refusés dans ces établissements et orientés vers les hôpitaux départementaux ou cantonaux ${ }^{13}$. L'exemple du dispensaire de Calmette est suivi dans d'autres villes en France. L'CEuvre de la tuberculose humaine, du docteur Samuel Bernheim, fonde entre 1901 et 1914 une vingtaine de dispensaires où est retenue l'idée d'assistance et d'éducation. Sous la présidence de la marquise de Ganay, philanthrope ${ }^{14}$, est par ailleurs créé en 1913 un comité d'infirmières visiteuses en France ${ }^{15}$.

\section{Principes généraux des dispensaires établis avant 1914}

7 Jusqu'à la veille de la Grande Guerre, les dispensaires donnent gratuitement des soins et apportent du secours à tous les indigents, à moindres frais et sans les séparer de leur famille ${ }^{16}$. On les trouve surtout dans les grandes agglomérations, et plus spécifiquement dans les quartiers populaires. Toutes les grandes villes françaises sont également équipées d'un dispensaire spécialisé dans la lutte contre la tuberculose. À Paris, les deux plus importants sont ceux de l'hôpital Beaujon et de l'hôpital Laënnec ${ }^{17}$. L'initiative est vraiment d'origine privée: les dispensaires sont mis en place dans le cadre d'œuvres de charité, portées le plus souvent par des médecins sensibilisés à ces questions. À Belleville par exemple, le dispensaire, complètement indépendant de l'Assistance publique de Paris, est fondé notamment grâce à des donations de médecins.

8 Ces premiers dispensaires sont d'assez grandes dimensions : ils prennent même parfois l'allure de petits équipements hospitaliers avec leurs nombreuses pièces où la séparation des sexes est la norme. En plus des salles de consultation, de multiples équipements sont installés afin d'assainir les corps : bains-douches, piscines, salles de gymnastique, salles de repos. De nombreuses ouvertures sont percées, pour aérer et faire entrer la lumière, comme dans celui de Saint-Maur-des-Fossés (Val-de-Marne), construit en $1904^{18}$ (fig. 2). La construction est réalisée avec des matériaux (meulières, briques, etc.) que l'on rencontre dans la plupart des édifices publics de cette époque, comme les mairies ou les écoles. Enfin, ces bâtiments sont décorés dans un style plutôt "régionaliste ", avec le jeu sur la polychromie des briques, l'encadrement des baies souligné d'archivolte et de claveaux de brique ou des pignons en façade. Les références à une architecture plus classique et "éclectique ", comme au Havre (Seine-Maritime) ou à Mulhouse ${ }^{19}$, affirment par ailleurs le rang des personnes qui financent le dispensaire. D'une manière générale, on note que le décor est très souvent riche pour des établissements accueillant des personnes socialement modestes. 
Figure 2

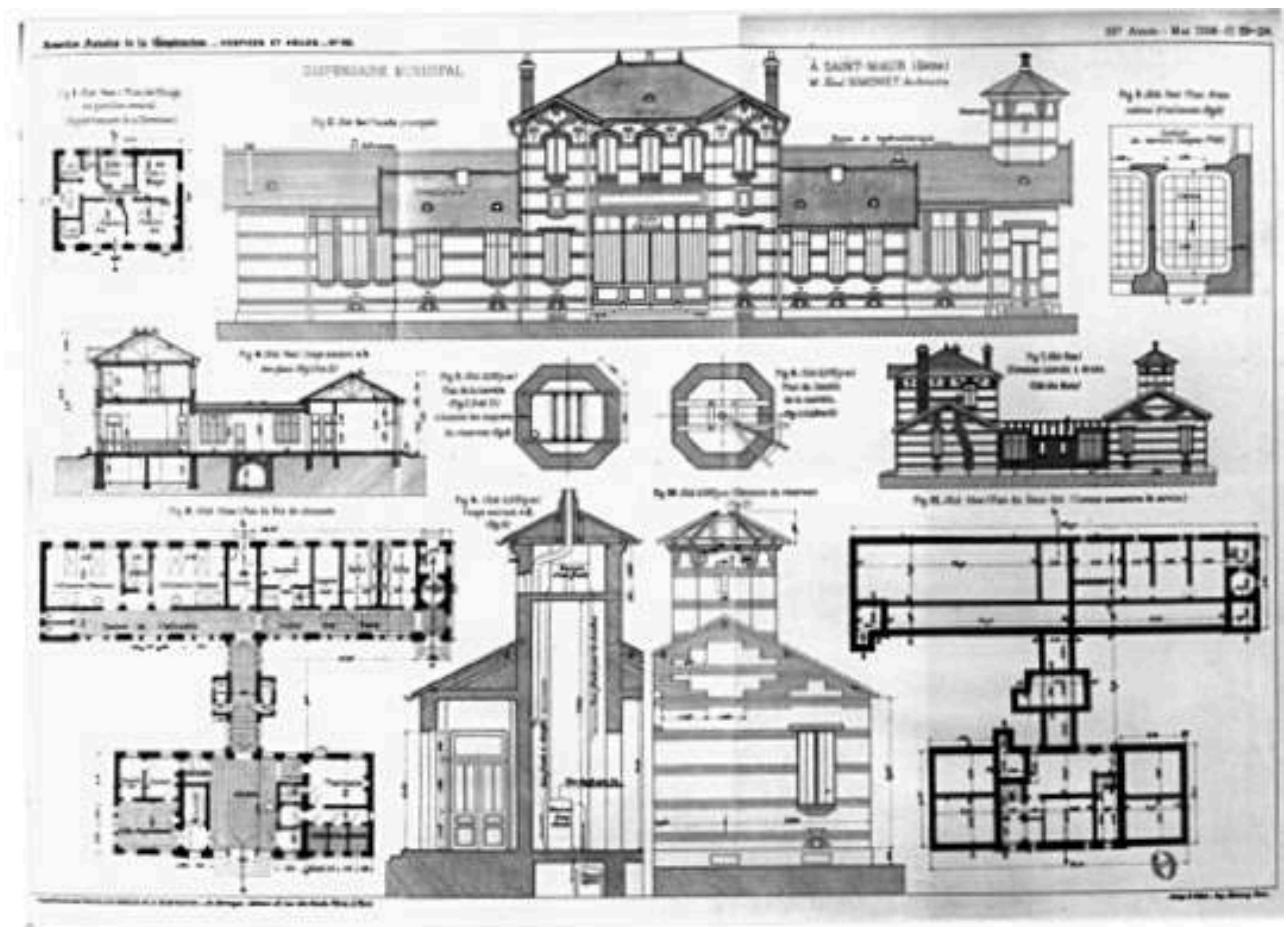

Plans, coupes, élévations du dispensaire de Saint-Maur-des-Fossés. Nouvelles annales de la construction, 1904.

Les dispensaires du Havre et de la rue des Pyrénées à Paris résument bien ces caractéristiques générales. En 1875, un premier dispensaire est établi au Havre ${ }^{20}$, au 43 de la rue Saint-Quentin, pour les enfants malades. Une étude sur les progrès de l'hygiène en France, réalisée entre 1878 et 1882, en souligne le caractère innovant ${ }^{21}$ :

Au congrès international d'hygiène de Paris en 1878, notre collègue M. le Dr Gilbert (du Havre) présentait un plan du dispensaire qu'il a fait élever dans cette ville et faisait en peu de mots comprendre son fonctionnement. Mettre entre les mains du médecin un outillage complet pour lui permettre de soigner toutes les maladies de l'enfance, supprimer la consultation externe qui ne donne que des résultats sans importance, qu'elle se fasse aux bureaux de bienfaisance ou dans les salles de consultation des hôpitaux, en les remplaçant par un traitement effectif, complet au dispensaire, voilà quelle a été l'idée du fondateur (... ${ }^{22}$.

Pour les auteurs de l'étude, c'est une des premières fois en France qu'un médecin peut mener des consultations en disposant dans l'établissement même de tout ce dont il besoin. Un deuxième dispensaire est élevé dans cette ville, rue du Rocher, entre 1895 et 1900 , et prend le nom de «dispensaire du docteur Gibert ». Il se caractérise par des façades de style éclectique, ponctuées de briques de différentes couleurs, des toitures à croupes, et une ornementation assez riche, surtout au niveau de l'encadrement des baies (fig. 3, fig. 4). 
Figure 3

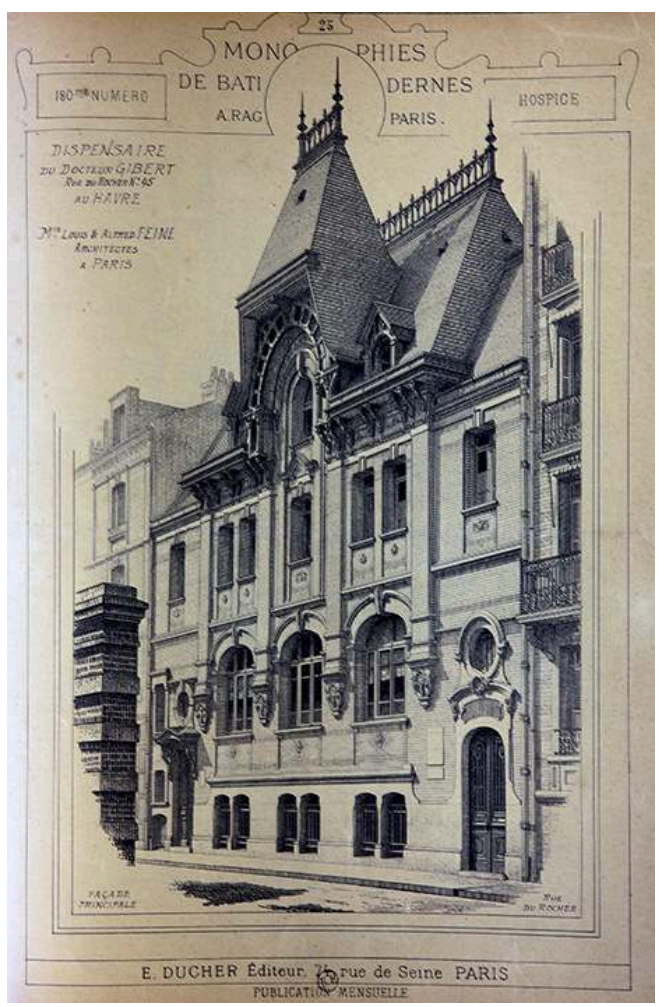

Façade principale du dispensaire du docteur Gilbert au Havre. Monographie de bâtiments modernes, $n^{\circ} 180$. 
Figure 4

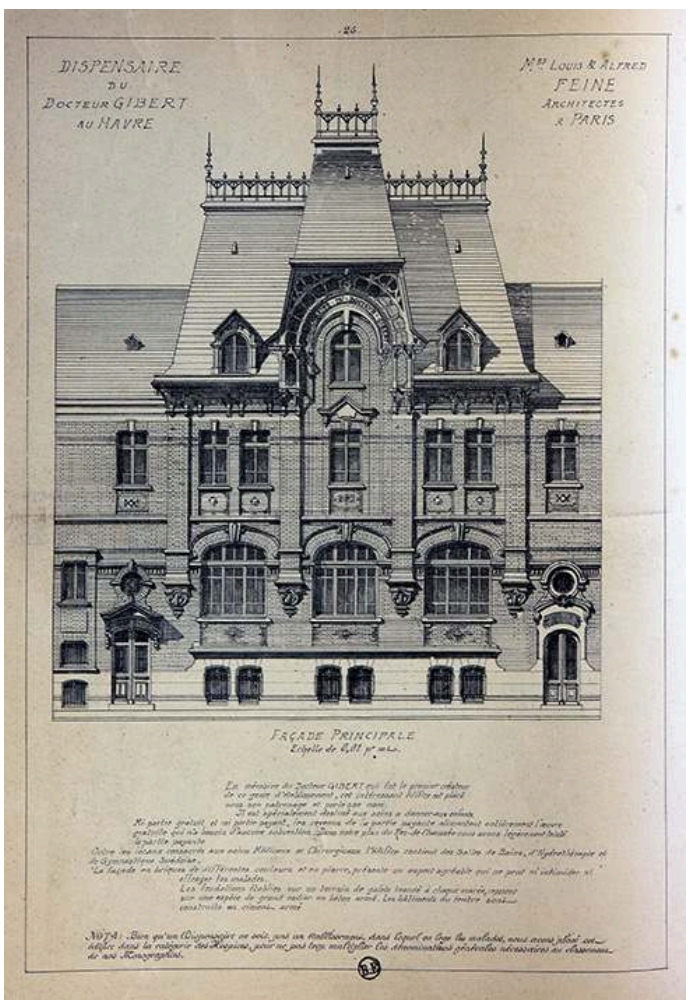

Façade principale du dispensaire du docteur Gilbert au Havre. Monographie de bâtiments modernes, $n^{\circ} 180$.

11 Sur le plan du bâtiment (fig. 5), on note une séparation entre les parties où les soins sont gratuits et celles où ils sont payants. Au sous-sol se trouvent les «bains-douches populaires » et une piscine, la natation étant considérée comme un excellent exercice pour les enfants. Le rez-de-chaussée se compose d'une entrée, d'une salle d'attente au centre, de salles de pansement et de chirurgie, de bains et de douches séparés garçons/ filles, et enfin d'une salle de gymnastique. 
Figure 5

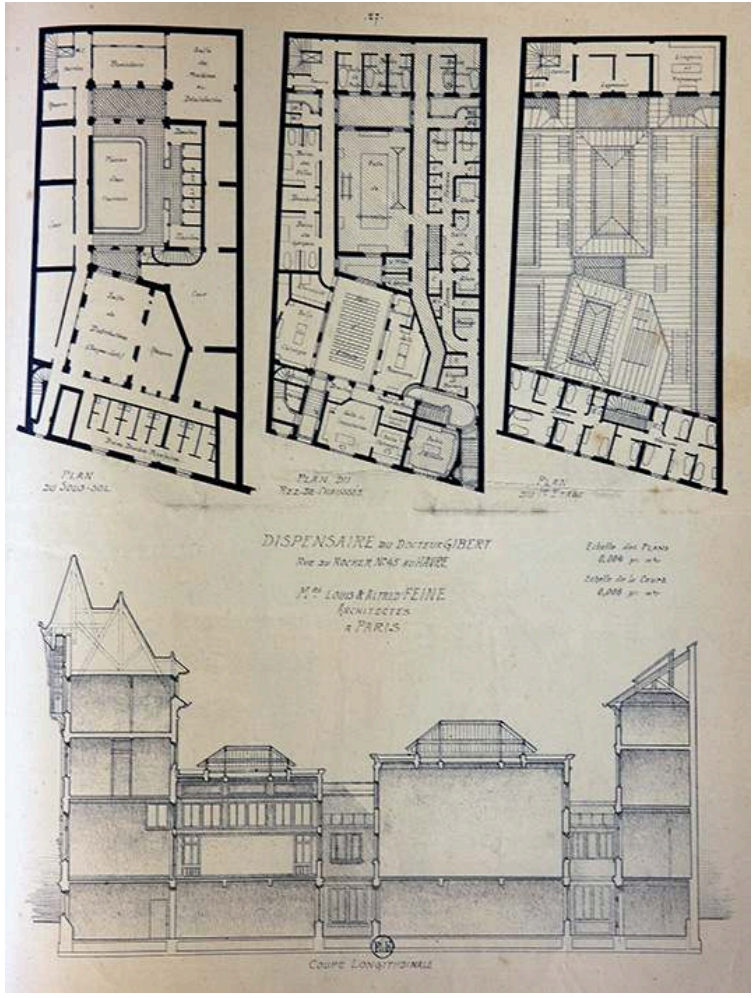

Plan du dispensaire du docteur Gilbert au Havre, Monographie de bâtiments modernes, $n^{\circ} 180$

12 Le dispensaire de la rue des Pyrénées (fig. 6), à Paris ${ }^{23}$, lui aussi appelé du nom de ses donateurs, Jouye, Rouve et Taniès ${ }^{24}$, est toujours visible depuis la voie publique, mais est inoccupé. Construit sur les plans ${ }^{25}$ de l'architecte Louis Bonnier ${ }^{26}$, il est typique des édifices publics de cette époque avec sa pierre meulière, sa toiture à croupes et ses nombreuses baies (fig. 7). 
Figure 6

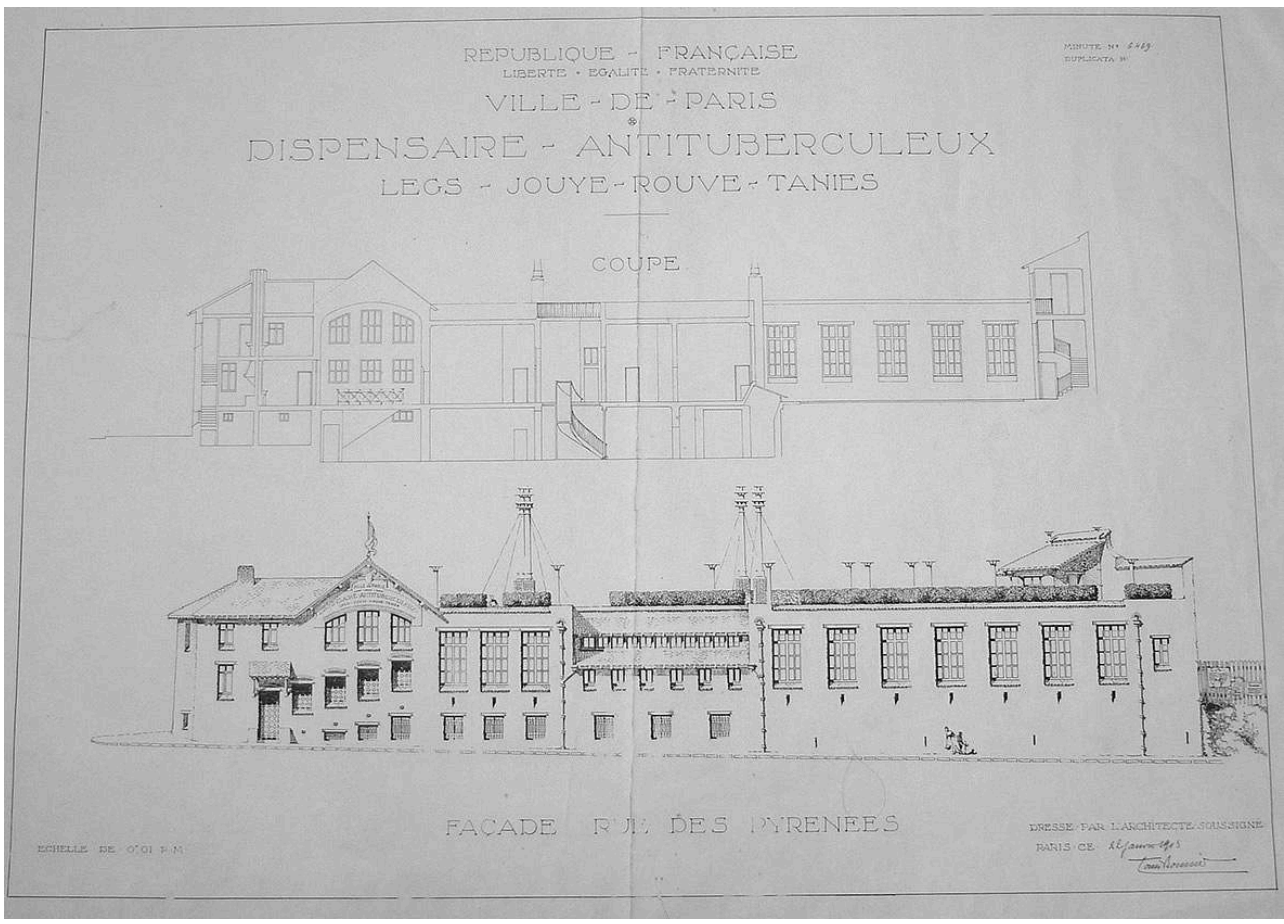

Façade principale du dispensaire situé à Paris, rue des Pyrénées, dessin de Louis Bonnier (architecte), 1905. Centre d'archives, Cité de l'architecture et du patrimoine, Réf. 035 Ifa 31.

(c) Centre d'archives, Cité de l'architecture et du patrimoine. 


\section{Figure 7}

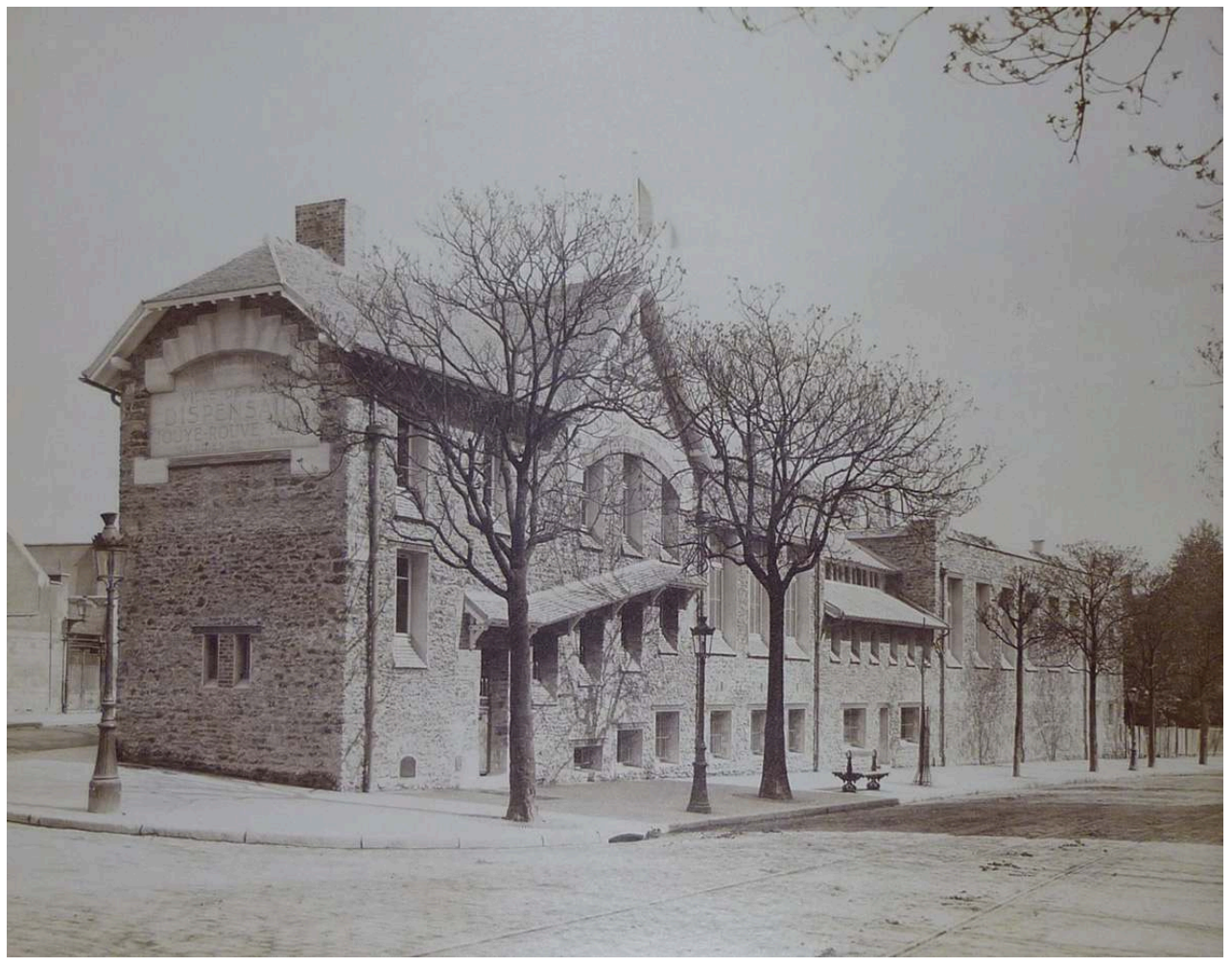

Dispensaire situé à Paris, rue des Pyrénées. Phot. Chevojon et Dufour, Centre d'archives, Cité de I'architecture et du patrimoine, Réf. 035 Ifa 508/1.

(c) Centre d'archives, Cité de l'architecture et du patrimoine.

\section{Figure 8}

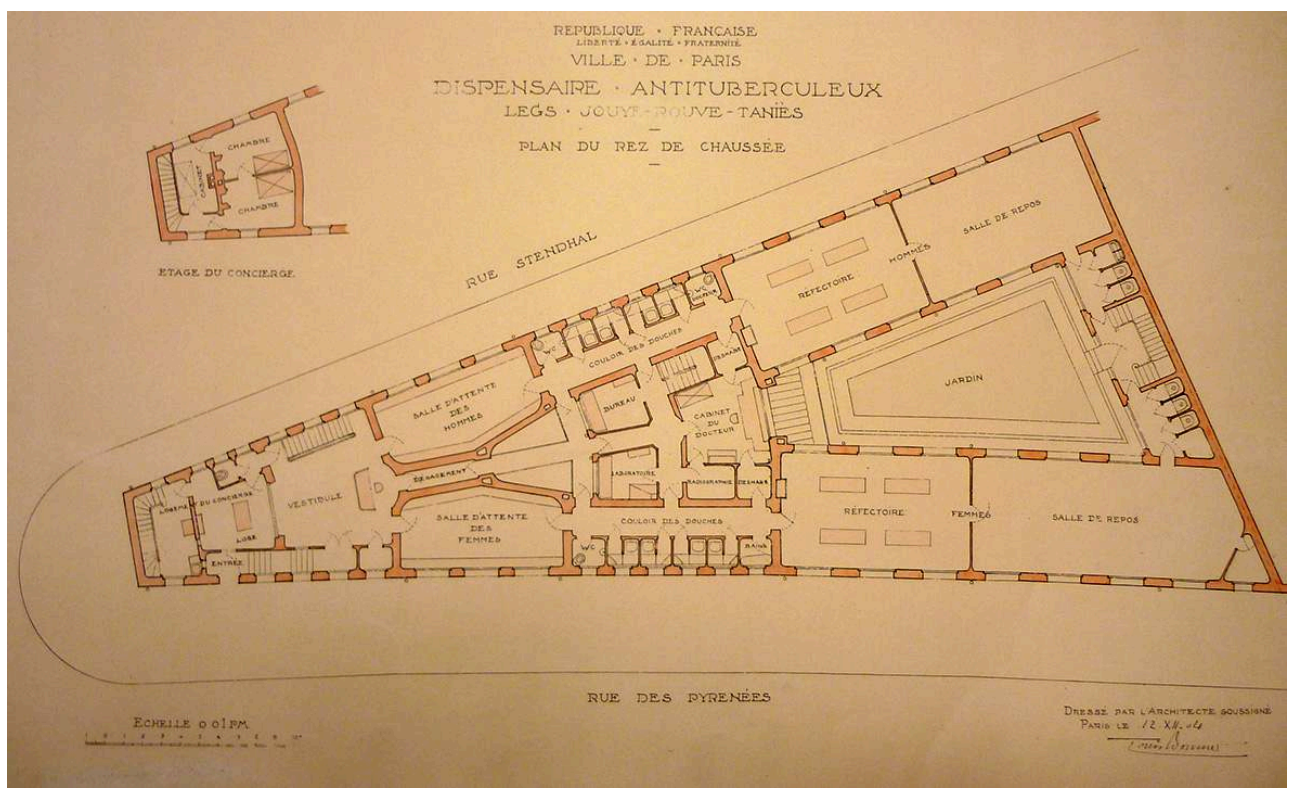

Plan du dispensaire situé à Paris, rue des Pyrénées. Centre d'archives, Cité de l'architecture et du patrimoine, Réf. 035 Ifa 31.

(c) Centre d'archives, Cité de l'architecture et du patrimoine. 
13 Le plan (fig. 8) est clairement organisé, de part et d'autre d'un jardin central, en fonction d'une séparation des sexes: ainsi y trouve-t-on deux salles d'attente, des douches, un laboratoire, le cabinet du médecin, des réfectoires et des salles de repos. Même les terrasses, situées aux niveaux supérieurs, sont distinctes (fig. 9, fig. 10).

\section{Figure 9}

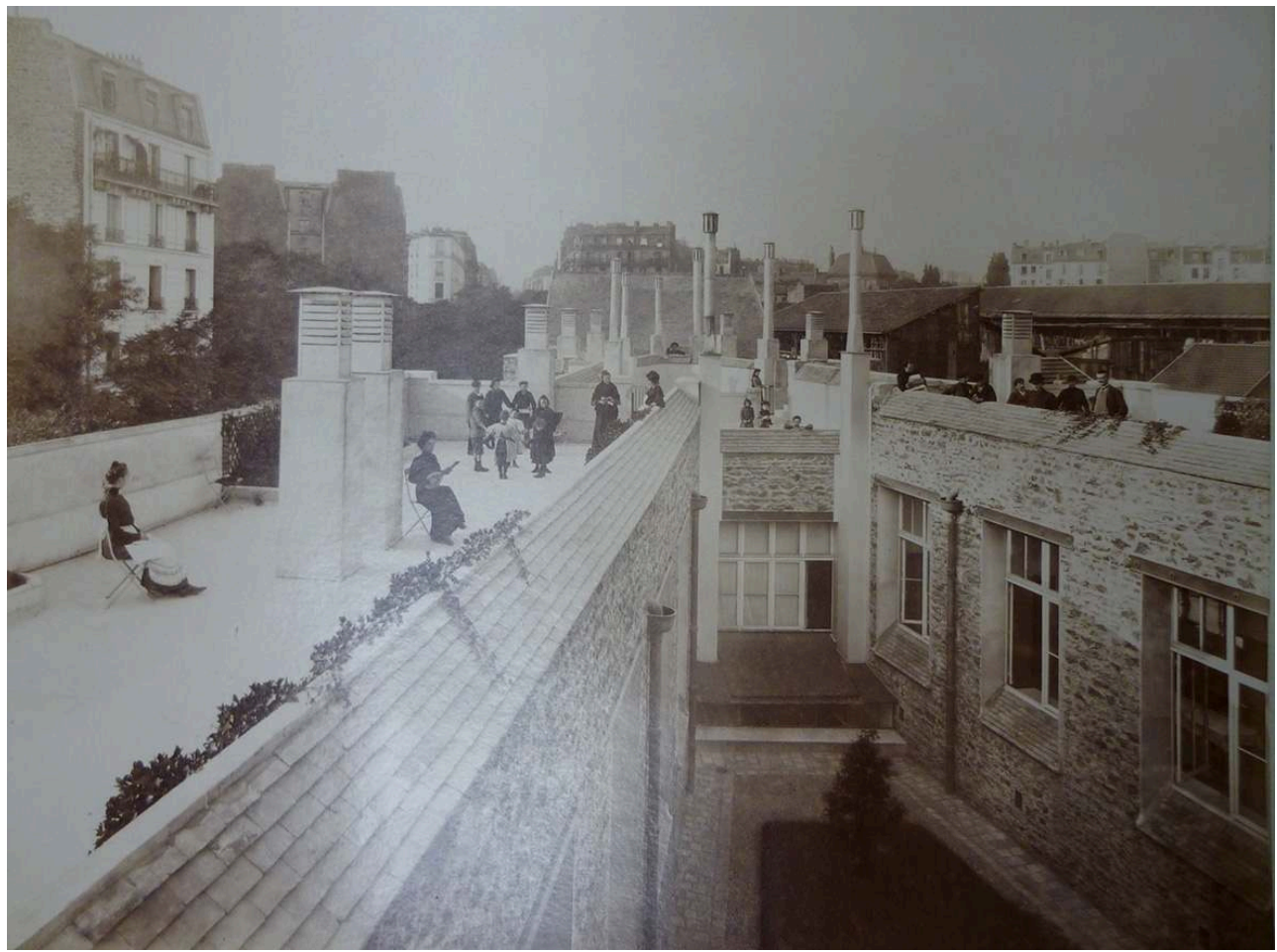

Vue du toit-terrasse du dispensaire situé à Paris, rue des Pyrénées. Phot. Chevojon et Dufour, Centre d'archives, Cité de l'architecture et du patrimoine, Réf. 035 Ifa 508/1.

(c) Centre d'archives, Cité de l'architecture et du patrimoine. 
Figure 10

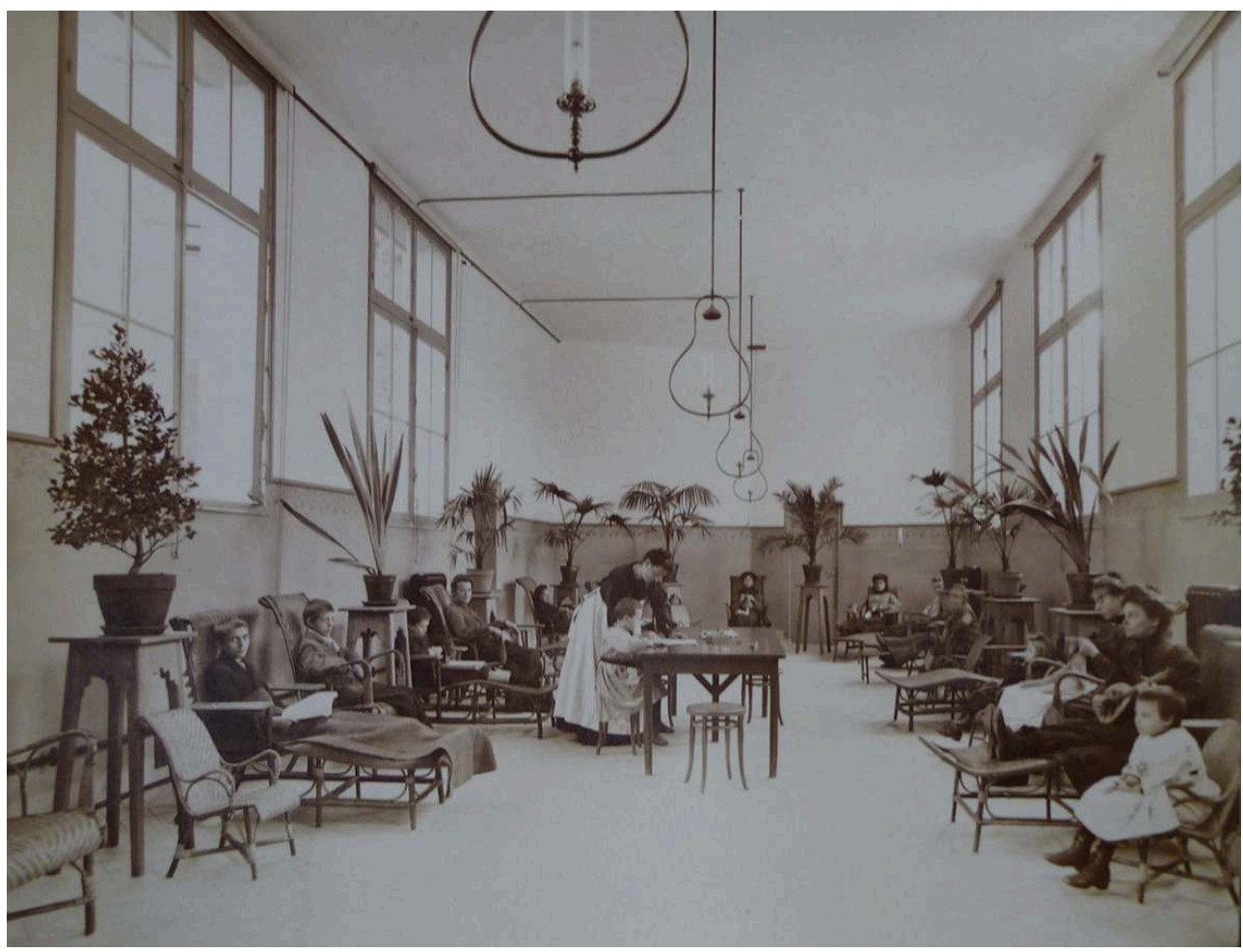

Vue d'une salle de repos réservée aux femmes et aux enfants, dispensaire situé à Paris, rue des Pyrénées. Phot. Chevojon et Dufour, Centre d'archives, Cité de l'architecture et du patrimoine, Réf. 035 Ifa 508/1.

(C) Centre d'archives, Cité de l'architecture et du patrimoine.

\section{La mise en place d'un réseau de dispensaires dans l'Entre-deux-guerres}

Entre 1906 et 1925, la France est passée du $5^{\mathrm{e}}$ au $2^{\mathrm{e}}$ rang mondial des pays les plus touchés par la tuberculose, qui sévit surtout dans les grandes agglomérations françaises, et plus précisément dans les zones industrielles et populaires. Devant l'alarmante contagion de la maladie parmi les soldats de la Grande Guerre, les pouvoirs publics prennent conscience des dangers qu'elle représente pour la défense nationale, mais aussi pour l'économie du pays.

C'est à la suite de cette prise de conscience que la loi Léon Bourgeois, du 15 avril 1916, impose, sur l'ensemble du territoire, la création de dispensaires d'hygiène publique. L'instauration, en janvier 1920, d'un ministère en charge de ces problèmes crée les conditions de leur multiplication avec la mise en œuvre d'une véritable politique d'hygiène sociale. 800 dispensaires résultant de cette loi sont recensés en $1936^{27}$.

\section{La prise de conscience des autorités publiques}

16 Cette lutte contre la tuberculose et la mortalité infantile s'inscrit dans un mouvement plus général de développement de l'action publique en matière sanitaire et sociale. Elle s'oriente notamment vers la suppression des taudis et des logements insalubres, la 
forte croissance des périphéries urbaines déplaçant l'activité édilitaire de l'Entre-deuxguerres vers les banlieues ${ }^{28}$.

L'objectif de la loi Bourgeois était de couvrir la France d'un réseau de dispensaires, bien distinct de celui des hôpitaux. Privilégiant l'action préventive, ces dispensaires publics ou privés faisaient l'objet de financements croisés provenant à la fois du ministère de tutelle, des conseils généraux et des communes, financements auxquels pouvaient s'ajouter des dons de particuliers. Le texte de loi précise que ces dispensaires devaient être "spécialement chargés de faire l'éducation antituberculeuse " ${ }^{29}$. C'est ainsi que tout un réseau d'institutions se met en place. En 1918, des offices départementaux d'hygiène sociale (article 10 de la loi Léon Bourgeois) sont organisés sous la direction des préfets pour créer des dispensaires, subventionner les cures et coordonner les œuvres privées. Un Office national est créé en 1919 pour les centraliser ${ }^{30}$ : association reconnue d'utilité publique en 1926, il est chargé par les pouvoirs publics de coordonner toutes les actions publiques et privées. Il bénéficie par la suite de nouvelles ressources financières publiques : en 1922, une taxe spéciale sur les cercles de jeux est instituée à son profit, ainsi qu'un prélèvement sur les recettes du Pari mutuel. Enfin, à partir de 1927, les campagnes nationales du timbre anti-tuberculeux sont lancées, associant ainsi toute la population à l'effort national ${ }^{31}$.

Petit à petit, la vaccination par le BCG, mise au point en 1927 par les docteurs Albert Calmette et Camille Guérin, conduit à confier aux dispensaires la lutte contre la mortalité infantile. Mais la généralisation de ce processus est lente et laborieuse face à la méfiance de certains médecins. Il faut attendre 1950 pour qu'elle devienne obligatoire dans les écoles ${ }^{32}$.

\section{Architectures et usages des dispensaires issus de la loi Bourgeois}

\section{Un modèle simple à mettre en œuvre}

En 1918, le Journal de la société statistique de Paris donne une définition assez concise de ces nouveaux équipements publics: "un dispensaire, anti-tuberculeux ou antisyphilique, ce sont quatre ou cinq pièces propres, aérées, éclairées, ripolinées " ${ }^{33}$. Cette simplicité d'organisation conduit à l'aménagement de nombreux dispensaires dans des édifices ayant à l'origine une autre fonction, comme des hôtels-Dieu ou des maisons d'habitation. On n'y note aucune caractéristique architecturale particulière, si ce n'est celles du bâtiment d'origine. Dans les petites agglomérations, de nombreux bâtiments spécialement dédiés à ce rôle de dispensaire sont par ailleurs construits. Ils suivent le modèle du pavillon s'élevant sur un ou deux niveaux et abritant quelques pièces pour la consultation. Les constructions sont réalisées avec les matériaux locaux les plus répandus comme la brique, le moellon ou la meulière, selon la région. Une certaine veine régionaliste est perceptible dans les élévations extérieures, comme au dispensaire de Juvisy-sur-Orge (fig. 11) (Essonne) construit en $1927^{34}$ ou à Loudéac (fig. 12) et Perros-Guirec (Côtes-d'Armor). Construit en 1930 sur les plans de Jean Fauny, alors architecte départemental des Côtes-du-Nord ${ }^{35}$, le dispensaire de Loudéac présente un plan carré ; il se compose d'un rez-de-chaussée percé d'une large baie en plein-cintre, et d'un étage de combles ménagé sous un toit à longs pans et à croupes, sur lesquels se greffent des faux-pignons ajourés et ornés de faux pans de bois. On peut signaler que ce modèle architectural fut plusieurs fois mis en œuvre par Fauny dans le département : les dispensaires d'Évran, Ploubalay ou Plestin-les-Grèves ${ }^{36}$ présentent les 
mêmes élévations. Le mouvement moderne n'est pour autant pas mis de côté, notamment dans les constructions en béton. Pavillons s'élevant sur deux étages en brique et en béton, les dispensaires de Beauchamp (Val-d'Oise) ou de Courbevoie ${ }^{37}$ (Hauts-de-Seine) répondent à cette deuxième catégorie.

Figure 11

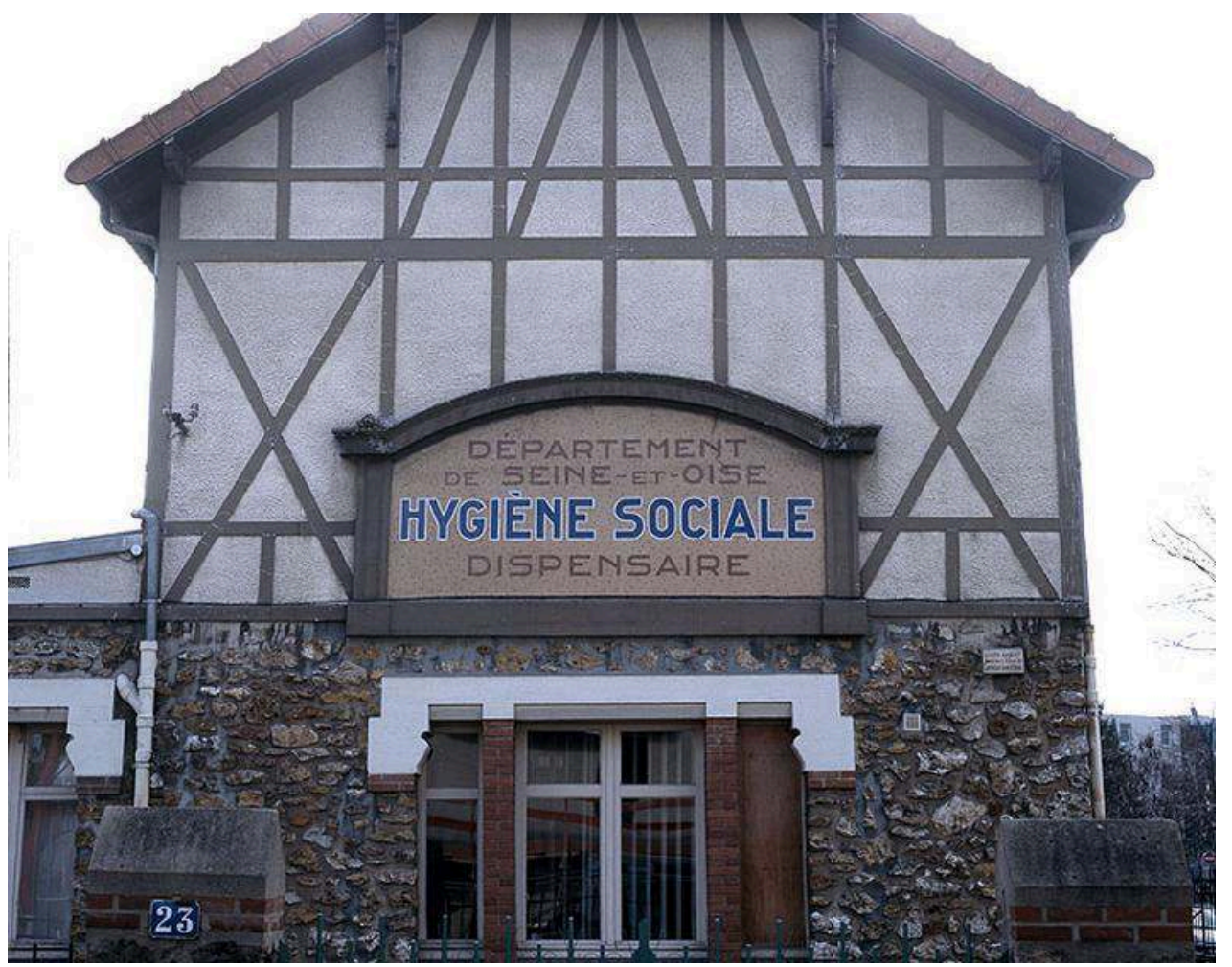

Façade-pignon du dispensaire de Juvisy-sur-Orge.

Phot. Ayrault, P., 2005. @ Région Île-de-France-Inventaire général du patrimoine culturel ; @ Conseil départemental de l'Essonne. 
Figure 12

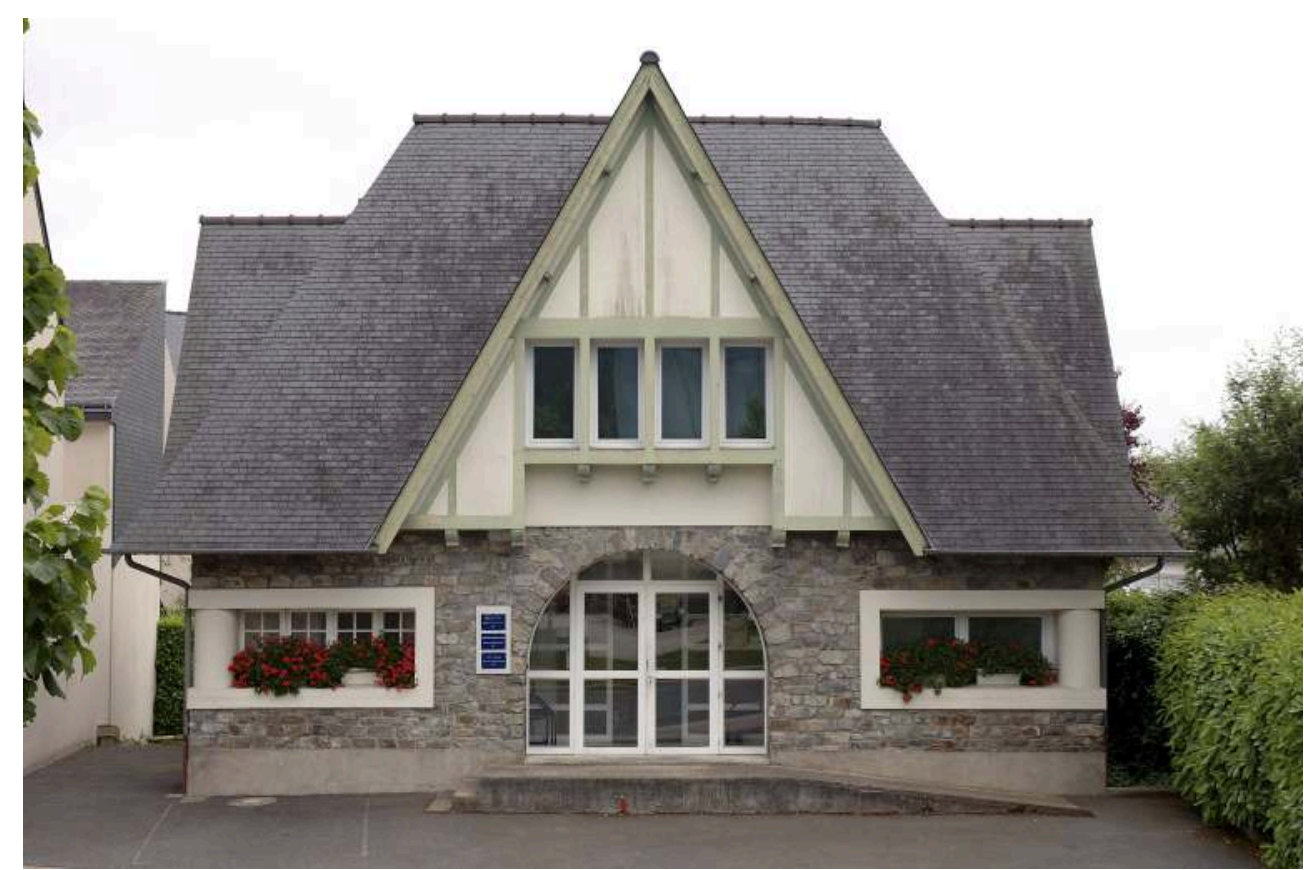

Façade principale du dispensaire de Loudéac.

Phot. Scheinkmann, X., 2005. ( I Inventaire général, ADAGP.

\section{Des principes d'organisation rationalisés et codifiés}

Les dispensaires, notamment les dispensaires anti-tuberculeux, se doivent de répondre à deux missions essentielles : l'accueil des patients et les visites à domicile par des infirmières "visiteuses $~_{38}$. En effet, "le rôle du dispensaire est de reconnaître la tuberculose, de classer les tuberculeux, de déterminer ceux qui sont curables et, pour chacun de ceux-ci, les modalités de la cure hygiénodiététique $»^{39}$. Les infirmières sont pour leur part un rouage nécessaire de la lutte antituberculeuse; ce sont elles qui surveillent les malades en dehors du dispensaire, veillent à l'application des mesures de prophylaxies, etc..$^{40} »$.

D'où l'adoption au niveau national de plans de dispensaire types, notamment dans les petites agglomérations. Ils sont généralement composés d'une salle d'accueil, où une infirmière établit un diagnostic préalable, et d'un cabinet médical, qui communique éventuellement avec une salle de pansement et d'opération pour des interventions bénignes (fig. 13). 


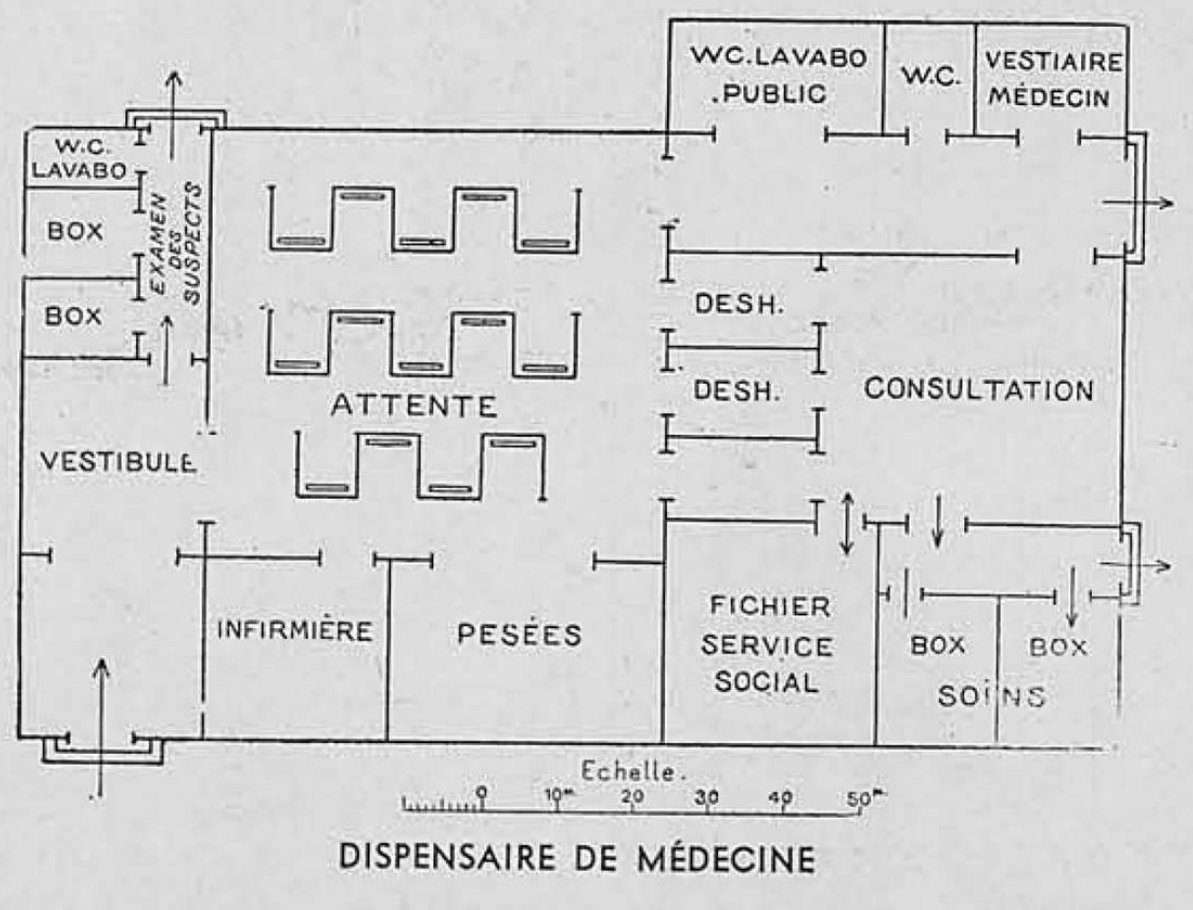

Plan d'un dispensaire municipal. L'Architecture d'aujourd'hui, 1934, n9, p. 62.

L'attention portée à l'hygiène (on y trouvait systématiquement des lavabos et des toilettes) et au risque de contagion des personnes examinées y était très grande. Reportons-nous à ce sujet à la description donnée par l'article de L'Architecture d'aujourd'hui ${ }^{41}$ en 1934 :

Le dispensaire médical comprend:

1) un box pour l'infirmière chargée de surveiller des consultants ; la situation de ce box permet à l'infirmière de surveiller également la salle d'attente et de communiquer facilement avec la salle des pesées, le fichier et la consultation ;

2) un service de triage à l'arrivée, comprenant des box pour les enfants suspects de maladies contagieuses, des lavabos et des w.-c.; ce service est parfaitement isolé ; les malades examinés et reconnus contagieux sortent directement ;

3) une salle d'attente qui est meublée de box bas, en métal, disposés en chicane ; une salle de propreté avec lavabos et w.-c. est à la disposition des consultants ;

4) une salle des pesées pouvant avoir une autre destination s'il ne s'agit pas d'une consultation pour enfants (laboratoire d'un dispensaire antivénérien, local pour soins dentaires, etc.) ;

5) une salle de consultation reliée directement : avec la salle d'attente par des box de déshabillage et un couloir; avec le fichier (fiches médicales et du service social) et avec une petite salle de traitement, elle-même divisée en box; après le traitement, les malades peuvent sortir directement de cette salle ;

6) un vestiaire personnel pour le médecin.

\section{Naissance de véritables équipements de santé au niveau local}

Certaines communes, notamment les plus importantes par le nombre d'habitants, conçoivent les dispensaires comme un véritable équipement public, au même titre que l'école, le cinéma, la piscine ou le centre sportif. La cité des Gautherets par exemple, aménagée près de Montceau-les-Mines (Saône-et-Loire), propose de nombreux 
équipements collectifs parmi lesquels on compte l'école de garçons, le dispensaire et la Goutte de lait. D'autres mettent même en place des ensembles médicaux-sociaux plus vastes. C'est le cas de Boulogne (Hauts-de-Seine), ville alors en pleine mutation face aux annexions parisiennes et à l'extension des quartiers industriels. Devant faire face à des problèmes sociaux croissants, la commune décide, en 1938, de faire construire un centre d'hygiène social ${ }^{42}$ par l'architecte de l'office des HBM de la Seine, Roger-Léopold Hummel ${ }^{43}$. Cet équipement devait réunir le dispensaire municipal, la polyclinique scolaire, le centre de consultation prénatale et celle des nourrissons, l'œuvre de la Goutte de lait, un service de psychologie pédiatrique ainsi qu'un centre de gymnastique orthopédique. On note enfin plusieurs exemples de dispensaires associés à des bainsdouches publics, comme à Vimoutiers (Orne, détruit) ${ }^{44}$ et à Alfortville (Val-de-Marne) ${ }^{45}$.

\section{Le dispensaire de Montceau-les-Mines : vers une première reconnaissance patrimoniale?}

\section{La Société des mines de Blanzy et Montceau-les-Mines}

C'est en 1833 que Jules Chagot fonde la Compagnie des mines de Blanzy, qui prend en 1856 la raison sociale Jules Chagot et Cie. En 1900 seulement, la Compagnie est transformée en Société anonyme des mines de Blanzy. En dehors de ses activités industrielles, la famille Chagot s'investit dans l'administration de Montceau-les-Mines, créée en 1856 et très vite surnommée «Chagot-ville ${ }^{46}$ ». En effet, pour assurer le développement de l'extraction, un personnel de plus en plus nombreux est recruté. Mais retenir ces nouveaux travailleurs à Montceau suppose que l'on puisse leur offrir des conditions de travail et de vie meilleures que celles proposées par d'autres établissements industriels ${ }^{47}$. Ce contrôle de l'espace et du temps des populations, tel qu'il est exercé à Montceau à la fin du XIX ${ }^{e}$ siècle et entre les deux guerres, repose sur quelques principes simples : des logements confortables, la moralité des ouvriers, un système scolaire efficace et des soins médicaux gratuits. Entre 1870 et 1890 , la Compagnie des mines divise le bassin minier en trois circonscriptions médicales, selon les aires géographiques définies par les puits d'extraction et les cités ouvrières. La Compagnie disposait d'un médecin-chef basé à l'hôpital, et de deux médecins en second chargés des visites à domicile ${ }^{48}$.

\section{L'établissement du dispensaire}

C'est dans ce contexte que la Société anonyme décide, dès 1922, d'ouvrir un " dispensaire antituberculeux » (fig. 14) géré par la Croix-Rouge ${ }^{49}$. Il est construit par l'architecte Marcel Fournier, architecte de la cité des Gautherets à Saint-Vallier, commune située près de Montceau. Ce dispensaire fait partie de tout un dispositif de défense antituberculeux mis sur pied en Saône-et-Loire par l'office départemental d'hygiène sociale. Financé par ce même office, par les subventions des communes avoisinantes et surtout, par celles de la Société anonyme ${ }^{50}$, il est placé sous la direction d'un médecin titulaire qui assure trois consultations par semaine, assisté de trois infirmières bénévoles. Entre 1922 et 1934, le dispensaire accueille plus de 24000 patients, essentiellement des mineurs. Plus de 1000 sont placés dans des 
sanatoriums ou des hôpitaux. Il dispense également des cours pour former des jeunes femmes au diplôme d'infirmière ${ }^{51}$.

Figure 14

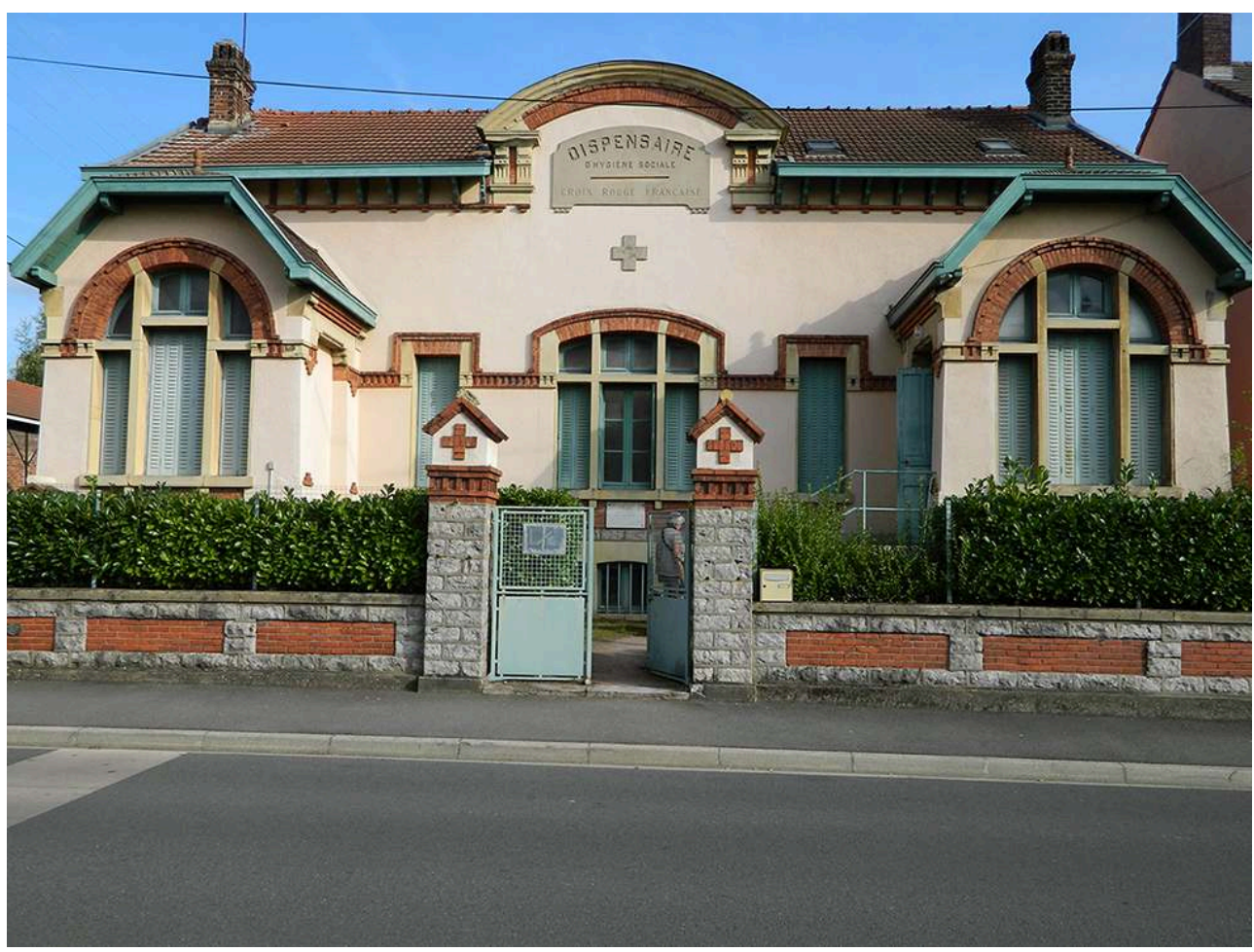

Façade principale du dispensaire de Montceau-les-Mines

Phot. Lestienne, C. 2011. @ Cécile Lestienne.

Du point de vue architectural, ce dispensaire synthétise tout ce qui a pu être présenté ci-dessus. Il s'agit d'un bâtiment au plan et à l'organisation assez simples. Élevé sur un seul niveau, il est percé de nombreuses ouvertures qui se rapprochent de la forme des baies dites «thermales ${ }^{52}$ ». Il est construit en moellon enduit avec un décor de brique assez soigné, et est couvert d'une toiture à croupes. Un fronton semi-circulaire porte la mention "Dispensaire d'hygiène sociale » : il est intéressant de retrouver un élément d'architecture assez savante et recherchée dans un petit dispensaire ouvrier. Ces caractéristiques architecturales ne sont pas nécessairement ce qui lui a valu son inscription au titre des monuments historiques en $2011^{53}$. L'importance de ce bâtiment dans l'histoire industrielle et sociale de Montceau-les-Mines, et dans celle, plus générale, de la condition ouvrière, semblait justifier sa protection.

Privés ou publics, monumentaux ou plus modestes, généraux ou anti-tuberculeux, les dispensaires ont quadrillé le territoire, au même titre que les mairies, les écoles ou les piscines. Pour Yvonne Knibiehler, ils n'ont sans doute pas été primordiaux, d'un point de vue médical, pour enrayer la tuberculose, dont la régression est surtout due à la vaccination, rendue obligatoire dans les écoles après la Seconde Guerre mondiale. Mais d'un point de vue "culturel», la lutte antituberculeuse a beaucoup appris aux médecins français en matière de propagande et de publicité. Enfin, d'un point de vue social, elle a mis en marche un processus irréversible, celui de la démocratisation de la médecine ${ }^{54}$. 


\section{NOTES}

1. - Nous reviendrons plus longuement sur ce sujet à la fin de cet article.

2. - Ces sources indispensables pour mener à bien l'histoire des dispensaires varient d'un département à l'autre. On signalera l'instrument de recherche établi et mis en ligne par les archives départementales d'Indre-et-Loire sur les fonds de l'Union départementale d'assistance aux tuberculeux (http://archives.cg37.fr/UploadFile/GED/SerieJ/1178540586.pdf).

3. - Les inventaires de ce fond sont en ligne, voir: http://archiwebture.citechaillot.fr/fonds/ FRAPN02_BAH [consulté le 28/11/2016].

4. - KNIBIEHLER, Yvonne. "La «lutte antituberculeuse " instrument de la médicalisation des classes populaires (1870-1930)». Annales de Bretagne et des pays de l'Ouest, t. 86, numéro 2, 1979, p. 321-336.

5. - CREMNITZER, Jean-Bernard. Architecture et santé. Le temps du sanatorium en France et en Europe. Paris : Picard, 2005 ; GRANDVOINNET, Philippe. Architecture thérapeutique. Histoire des sanatoriums en France (1900-1945). Genève : MetisPresses, 2014 ; MOREL, Marie-France. « Soigner le tout-petit : histoire de la puériculture et de la pédiatrie ». Dans NOBÉCOURT, Mathilde (dir.). La Naissance: histoire, cultures et pratiques d'aujourd'hui. Paris : Albin Michel, 2010, p. 172-189.

6. - Ministère de la Culture et de la Communication. Thésaurus de la dénomination architecturale et des espaces aménagés, 2011. Voir le site: http://data.culture.fr/thesaurus/page/ark:/67717/ T96-290 [consulté le 28/11/2016].

7. - Voir plus bas, note 8 .

8. - KNIBIEHLER, Yvonne. Art. cit.

9. - JULIEN, Pierre. «Pédiatrie sociale. Le créateur de la Goutte de lait et ses biberons : les biberons du Docteur Duffour ». Revue d'histoire de la pharmacie, 1997, vol. 85, n 315, p. 348-350, et SAUTEREAU, Anne. «Aux origines de la pédiatrie moderne : le docteur Léon Dufour et l'œuvre de la «Goutte de lait» (1894-1928)». Annales de Normandie, vol. 41, n 3, p. 217-233. Voir le site : http://www.persee.fr/doc/annor_0003-4134_1991_num_41_3_1889 [consulté le 28/11/2016].

10. - LAGRANGE, Frédéric. La société des mines de Blanzy en 1927. Blanzy : association « La mine et les hommes », 1996.

11. - KNIBIEHLER, Yvonne. Art. cit.

12. - Ibid.

13. - Introduction de l'instrument de recherche mis en place par Armande Le Roux sur le fonds $145 \mathrm{~J}$ de l'UDAT (Union départementale d'Assistance aux tuberculeux), archives départementales d'Indre-et-Loire, 2006. Voir ci-dessus note 2.

14. - Berthe de Béhague (1868-1930).

15. - KNIBIEHLER, Yvonne. Art. cit.

16. - Ibid.

17. - Ibid.

18. - Dossier d'inventaire établi en 1986 par Marie-Agnès Férault, $n^{\circ}$ IA00059545.

19. - Voir le dossier d'inventaire : http://www.culture.gouv.fr/documentation/memoire/HTML/ IVR42/IA00096507/index.htm.

20. - Voir le dossier d'inventaire établi en 1991 par Claire Étienne : IA00130468.

21. - NAPIAS, Henri et MARTIN, André-Justin. L'étude et les progrès de l'hygiène en France, de 1878 à 1882. Paris : G. Masson, 1882, p. 318. Nos remerciements vont à Pierre-Louis Laget qui nous a indiqué cette source.

22. - Ibid.

23. - Ce bâtiment est inscrit sur la liste de protection de la Ville de Paris et abrite, depuis 1984, le Service social départemental polyvalent $\mathrm{du} \mathrm{xx}^{\mathrm{e}}$ arrondissement (SSDP20). Les locaux ont fait 
l'objet de réaménagements intérieurs, par l'agence d'architecture Itar, entre 2012 et 2014 . Voir article dans Le Moniteur: http://www.lemoniteur.fr/article/du-sanatorium-au-bureau-unerehabilitation-atypique-a-paris-xxe-24360893?24361864=24361334\#24361864 [consulté le 28/11/2016].

24. - Le nom de ces donateurs apparaît dans le fonds Louis Bonnier du Centre des archives de la Cité de l'architecture et du patrimoine, ainsi que sur le bâtiment lui-même. Nous n'avons trouvé aucune information sur ces personnes.

25. - Des élévations, des plans et des photographies de ce dispensaire sont conservés dans le fonds de l'architecte Louis Bonnier, O 35 Ifa, Centre des archives, Cité de l'architecture et du patrimoine.

26. - Louis Bonnier (1856-1946), architecte de la Ville de Paris. Il est notamment l'auteur de la villa André Gide (Paris, $16^{\mathrm{e}}$ arr.) ou de la piscine de la Butte-aux-Cailles (Paris, $13^{\mathrm{e}}$ arr.).

27. - CREMNITZER, Jean-Bernard. Architecture et santé. Op. cit.

28. - MONNIER, Gérard (dir.). L'architecture moderne en France, 1889-1940. t. 1, 1889-1940. Paris : Picard, 1997.

29. - KNIBIEHLER, Yvonne. Art. cit.

30. - Ibid.

31. - Introduction de l'instrument de recherche mis en place par Armande Le Roux sur le fonds $145 \mathrm{~J}$ de l'UDAT, voir note 2.

32. - Ibid.

33. - «Le bilan de la tuberculose et de la syphilis en France ». Journal de la société statistique de Paris, 1918, t. 59.

34. - LE BAS, Antoine. Juvisy-sur-Orge, un territoire, des réseaux. Lyon : Éditions Lieux-dits (Cahiers du patrimoine), 2007. Voir aussi le dossier d'inventaire établi en 2003 par Antoine Le Bas : http:// www.culture.gouv.fr/documentation/merimee/PDF/sri11/IA91000672.pdf.

35. - Dossier d'inventaire établi par Jean-Pierre Ducouret en 2005, n ${ }^{\circ}$ IA22003652 (http:// patrimoine.region-bretagne.fr/gertrude-diffusion/dossier/dispensaire-actuellement-cabinetmedical/0ae1ce79-2fe2-4a8b-9544-6762336a206d).

36. - Dossiers d'inventaire $\mathrm{n}{ }^{\circ} \mathrm{IA} 22017344$ (http://patrimoine.region-bretagne.fr/gertrudediffusion/dossier/dispensaire-evran/d23f0ab1-2c31-4086-b827-de393d3b2cc3), IA22017373 (http://patrimoine.region-bretagne.fr/gertrude-diffusion/dossier/ancien-dispensaireactuellement-centre-de-la-solidarite-departementale/a8b7e798-30e6-40e3-96e8-a07e22f1ba14), et IA22003268 (http://patrimoine.region-bretagne.fr/gertrude-diffusion/dossier/dispensaire/ 23f26660-91ba-4c26-9c43-d655e8928af3), établis par Véronique Orain et Patrick Pichouron entre 2004 et 2010.

37. - Voir le dossier d'inventaire : http://www.culture.gouv.fr/documentation/memoire/HTML/ IVR11/IA00129946/index.htm.

38. - DESSERTINE, Dominique. «Les infirmières-visiteuses de la lutte antituberculeuse en France (1900-1930) ». Bulletin du Centre Pierre Léon d'histoire économique et sociale, 1995, p. 55-63.

39. - « Le bilan de la tuberculose et de la syphilis en France ». Art. cit.

40. - Ibid.

41. - «Dispensaires pour petites agglomérations ». L'Architecture d'aujourd'hui, 1934, nº 9, p. 62. Voir article en ligne sur le site de la Cité de l'architecture et du patrimoine: http:// portaildocumentaire.citechaillot.fr/pdfjs/web/viewer.html?file=/Infodoc/ged/

viewPortalPublished.ashx?eid\%3DIFD_FICJOINT_FRAPN02_AA_1934_09_12_PDF_1 [consulté le 28/11/2016].

42. - Voir le dossier d'inventaire : http://www.culture.gouv.fr/documentation/memoire/HTML/ IVR11/IA00119893/index.htm.

43. - LE BAS, Antoine. Boulogne-Billancourt, 1800-2000. Ville d'art et d'essai. 1800-2000. Paris/BoulogneBillancourt: APIF/Ville de Boulogne-Billancourt (coll. Images du patrimoine), 1997. Voir aussi le 
dossier d'inventaire $\mathrm{n}^{\circ}$ IA00119893 établi par Antoine Le Bas entre 1992 et 1993: http:// www.culture.gouv.fr/documentation/memoire/HTML/IVR11/IA00119893/index.htm.

44. - Dossier d'inventaire établi par Hélène Mousset en 1992, ${ }^{\circ}{ }^{\circ}$ IA00120182: http:// www.culture.gouv.fr/documentation/memoire/HTML/IVR25/IA00120182/INDEX.HTM.

45. - Dossier d'inventaire $\mathrm{n}^{\circ} \mathrm{IA00130074}$ établi par Véronique Belle en 1995 : http:// www.culture.gouv.fr/documentation/memoire/HTML/IVR11/IA00130074/index.htm.

46. - LAGRANGE, Frédéric. Chagot-ville ou La naissance de Montceau-les-Mines, 1851-1856-1881. Blanzy : association « La mine et les hommes », 2005.

47. - NOIRIEL, Gérard. « Du patronage au paternalisme: la restructuration des formes de domination de la main-d'œuvre ouvrière dans l'industrie métallurgique française ». Le Mouvement social, $1988, \mathrm{n}^{\circ} 144$.

48. - Dossier d'inventaire $\mathrm{n}^{\circ} \mathrm{IA71000037}$ établi par Frédéric Pillet en 1999: http:// www.culture.gouv.fr/documentation/merimee/PDF/sri26/IA71000037.PDF.

49. - Dossier d'inventaire $\mathrm{n}{ }^{\circ} \mathrm{IA71000070}$ établi par Frédéric Pillet en 1999: http:// www.culture.gouv.fr/documentation/merimee/PDF/sri26/IA71000070.PDF.

50. - Ces informations ont pu être réunies grâce à la consultation d'archives conservées au centre de la Croix-Rouge française, à Montceau-les-Mines. Ces archives ne sont cependant pas classées.

51. - Ibid.

52. - Voir PÉROUSE DE MONTCLOS, Jean-Marie. Principes d'analyse scientifique. Architecture: méthode et vocabulaire. Paris : Éditions du patrimoine/Centre des monuments nationaux, $6^{\mathrm{e}}$ éd., 2007 ( $1^{\mathrm{e}}$ éd. 1972) : «Fenêtre thermale : dans l'architecture antique, classique et moderne, fenêtre en forme de demi-cercle posé sur son diamètre, divisée par deux meneaux », p. 192.

53. - Ce dispensaire a été inscrit au titre des monuments historiques en 2011. Dès 2009, l'ancien dispensaire ainsi que les écoles maternelle et primaire de la cité $\mathrm{n}^{\circ} 11$ de la Compagnie des mines de Lens avaient fait l'objet d'une protection d'ensemble. Il s'agissait des éléments les plus représentatifs, et encore en place, de cette cité élevée au début du xxe siècle, voir :http:// www.culture.gouv.fr/public/mistral/merimee_fr.

54. - KNIBIEHLER, Yvonne. Art. cit.

\section{RÉSUMÉS}

Le développement des dispensaires à la fin du XIX ${ }^{\mathrm{e}}$ siècle procède d'une double préoccupation : la mortalité infantile, qui ne cesse de croitre, et les ravages de la tuberculose dans les milieux populaires. Par ailleurs, l'alarmante contagion de la maladie parmi les soldats de la Grande Guerre fait prendre conscience aux pouvoirs publics des dangers qu'elle représente pour la défense nationale, mais aussi pour l'économie du pays. C'est la loi dite «Léon Bourgeois ", du 15 avril 1916, qui impose sur l'ensemble du territoire la création de dispensaires d'hygiène publique. Financés par les communes, les industriels ou les sociétés de secours mutuel, ils sont destinés à l'origine aux plus démunis, le plus souvent dans une situation sanitaire critique, avant d'être ouverts à tous dans le cadre d'une politique d'hygiène publique. À partir d'exemples détaillés, cet article s'attache à examiner les caractéristiques architecturales de ces bâtiments et leur évolution.

The development of dispensaries at the end of the nineteenth century was based on a double preoccupation: infant mortality, which did not stop rising, and the ravages of tuberculosis 
amongst the poor. The alarming contagion of this illness amongst the soldiers of the First World War also alerted the authorities to the dangers it presented for national defence and for the country's economy. The so-called Léon Bourgeois law of 15 April 1916 made the creation of dispensaries of public hygiene throughout the national territory an obligation. Financed by local authorities, by industrial patrons or by mutual aid associations, they were originally intended for the poorest sections of the population, often is dire sanitary conditions, before being opened to all in a context of a national policy of public health. Based on the detailed examination of a selection of examples, this article examines the architectural characteristics of the buildings and how these evolved.

INDEX

Mots-clés : dispensaire, "Goutte de lait », Léon Bourgeois, tuberculose, mortalité infantile Keywords : dispensary, ‘Goutte de lait' (milk clinics), Léon Bourgeois, tuberculosis, infant mortality

\section{AUTEUR}

\section{CÉCILE LESTIENNE}

Attachée de conservation du patrimoine, Responsable de l'Inventaire du patrimoine, archives départementales du Val-d'Oise cecile.lestienne@valdoise.fr 